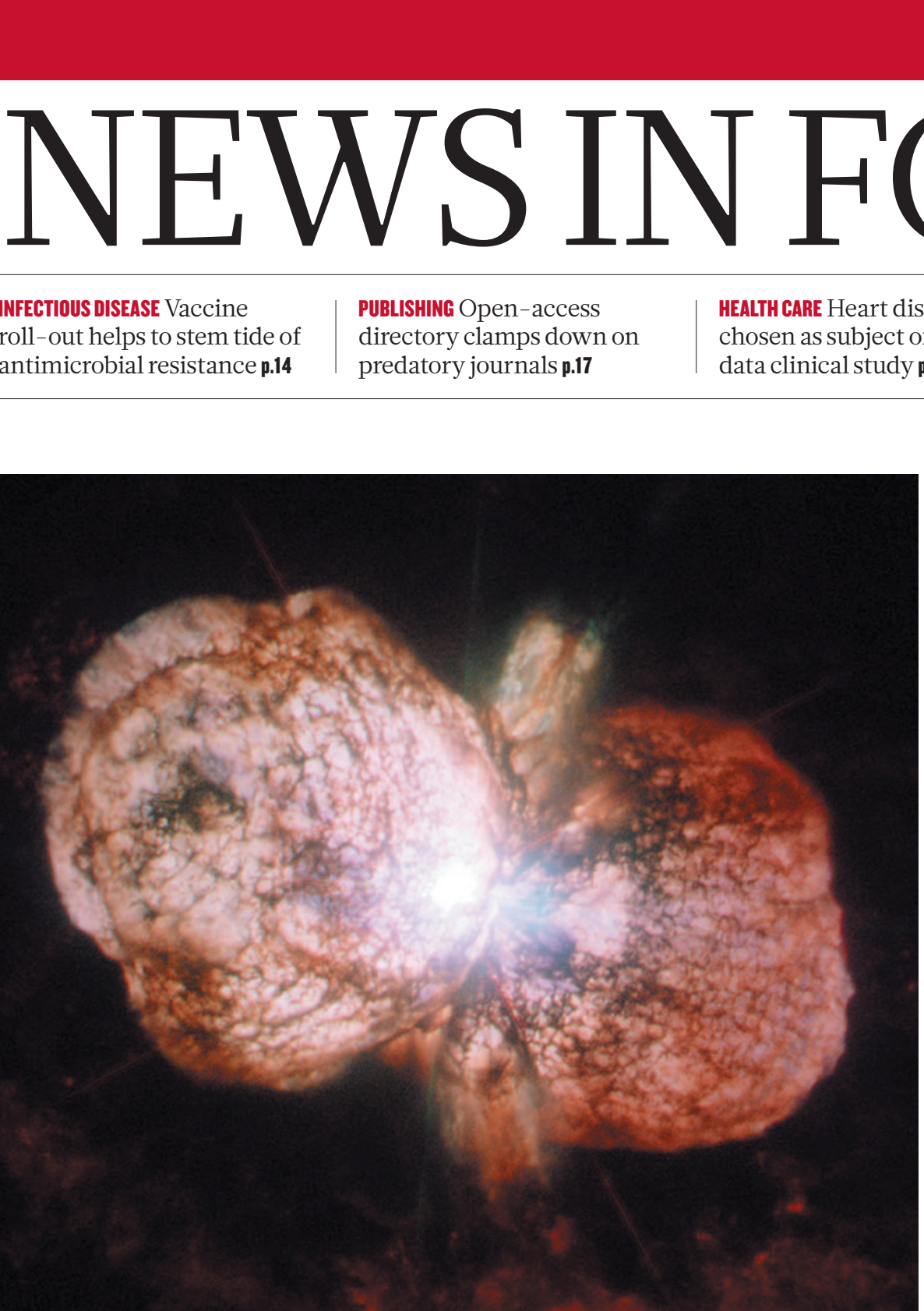

The massive binary-star system $\eta$ Carinae resembles the first stars that formed in the early Universe.

\title{
ASTRONOMY
}

\section{Binary star to spill celestial secrets}

\section{Close approach and violent interaction of stars in $\eta$ Carinae system will provide rare insight into stellar enigma.}

\section{BY ALEXANDRA WITZE}

A fter centuries of perplexing scientists with its wildly erratic behaviour, a nearby star may give up some of its secrets in the next couple of weeks.

A binary system, $\eta$ Carinae has two stars that swing past one another every 5.5 years. The
Astronomers are watching the show in the hope of learning what drives this enigmatic system. In the 1840s, $\eta$ Carinae had a mysterious eruption; in recent decades, it has again brightened unexpectedly (see 'Stellar show'). "The star is in an awfully deranged state, and no one knows why," says Kris Davidson, an astronomer at the University of Minnesota in Minneapolis.

Some answers may come in the next few weeks. Theoretical work suggests that when $\eta$ Carinae's secondary star passes by, its fast stellar winds bore a huge hole into the outer layers of the primary star (T. I. Madura et al. Mon. Not. R. Astron. Soc. 436, 3820-3855; 2013). Astronomers expect that if they are right about this, then a specific series of events will unfold this month - including a quick rise in the system's X-ray production after a drop that began in mid-July.

Studying $\eta$ Carinae has implications far beyond understanding one peculiar celestial system. Uncovering its secrets could help researchers to better understand the earliest stars that winked into existence. $\eta$ Carinae is similar in mass to the first stars that formed in the Universe, billions of years ago. Most of today's stars are much lighter, so $\eta$ Carinae is a rare modern example of how such a massive star might operate - at the highly observable distance of 2,300 parsecs from Earth.

Across the Southern Hemisphere, professional and amateur astronomers are pointing their telescopes at the star, in the constellation Carina. "It's the biggest effort ever," says Theodore Gull, an astrophysicist at NASA's Goddard Space Flight Center in Greenbelt, Maryland.

No one knows exactly when or how $\eta$ Carinae's companion will make its closest approach, but by mid-August it is likely to pass the primary at a distance equivalent to that between Mars and the Sun. Both stars in $\eta$ Carinae emit powerful stellar winds, which at close range collide, producing a 'bow shock' like that seen in front of ships. The mutual tangle sets off a sequence of bizarre events.

The stars began brightening in the visible part of the electromagnetic spectrum in April and then again in a sharper peak beginning in mid-June - probably as the companion star approached and began interacting with the primary star's winds, says Eduardo Fernández Lajús, an astronomer at the National University of La Plata in Argentina. The system's bigger star - some 90 times the mass of the Sun - is incredibly unstable, always seemingly on the verge of blowing up. When the smaller companion star makes its closest approach to the primary star, as is happening now, the interaction between the two triggers violent changes in the high-energy radiation pouring out of the system. 


\section{STELLAR SHOW}

Scientists have struggled to explain the erratic behaviour of the binary star $n$ Carinae, which brightened unexpectedly in the 1840s and again more recently.

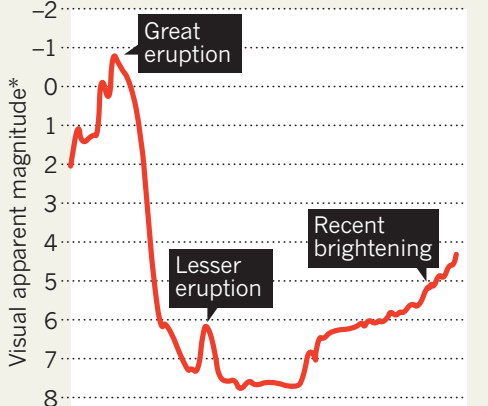

$\begin{array}{llllll}1820 & 1860 & 1900 & 1940 & 1980 & 2020\end{array}$ * Lower numbers represent greater brightness.

X-ray production peaked in mid-July and has since plummeted to near zero probably as the colliding winds, where the X-rays are born, have become entirely unstable and collapsed.

The Hubble Space Telescope and other instruments are also tracking dramatic changes in the chemical-element signatures found in $\eta$ Carinae's light spectrum. The interaction between the two approaching stars can strip electrons from elements such as iron and helium, ionizing them more strongly than in normal celestial environments. "You have these bare helium nuclei — that's awfully hard to make in normal circumstances," says Gull. Watching this process over time helps to reveal how the stellar winds are interacting.

At the Pico dos Dias Observatory in southern Brazil, astronomer Augusto Damineli has been spending every night since 25 July trying to catch a glimpse of $\eta$ Carinae through the winter clouds. On 29 July, his team finally caught a brief opening and managed to gather data showing that a helium spectral line is dropping in just the pattern that Damineli expected. "TOUCHDOWN!" he wrote in an e-mail.

In 2009, when $\eta$ Carinae had its most recent close encounter, the system's X-ray production plunged and then shot back up in half the time it did in 2003. That could be because the primary star's winds are slowing down, so it takes less time for the whole system to recover. If the wind speeds have continued to drop, X-ray emissions might shoot up even faster than last time.

Seeing such big differences from one close encounter to the next is "what everyone is waiting for", says Andrea Mehner, an astronomer at the European Southern Observatory in Santiago, Chile, who is monitoring $\eta$ Carinae with Hubble. "We cannot make the star do something exciting if it doesn't want to."

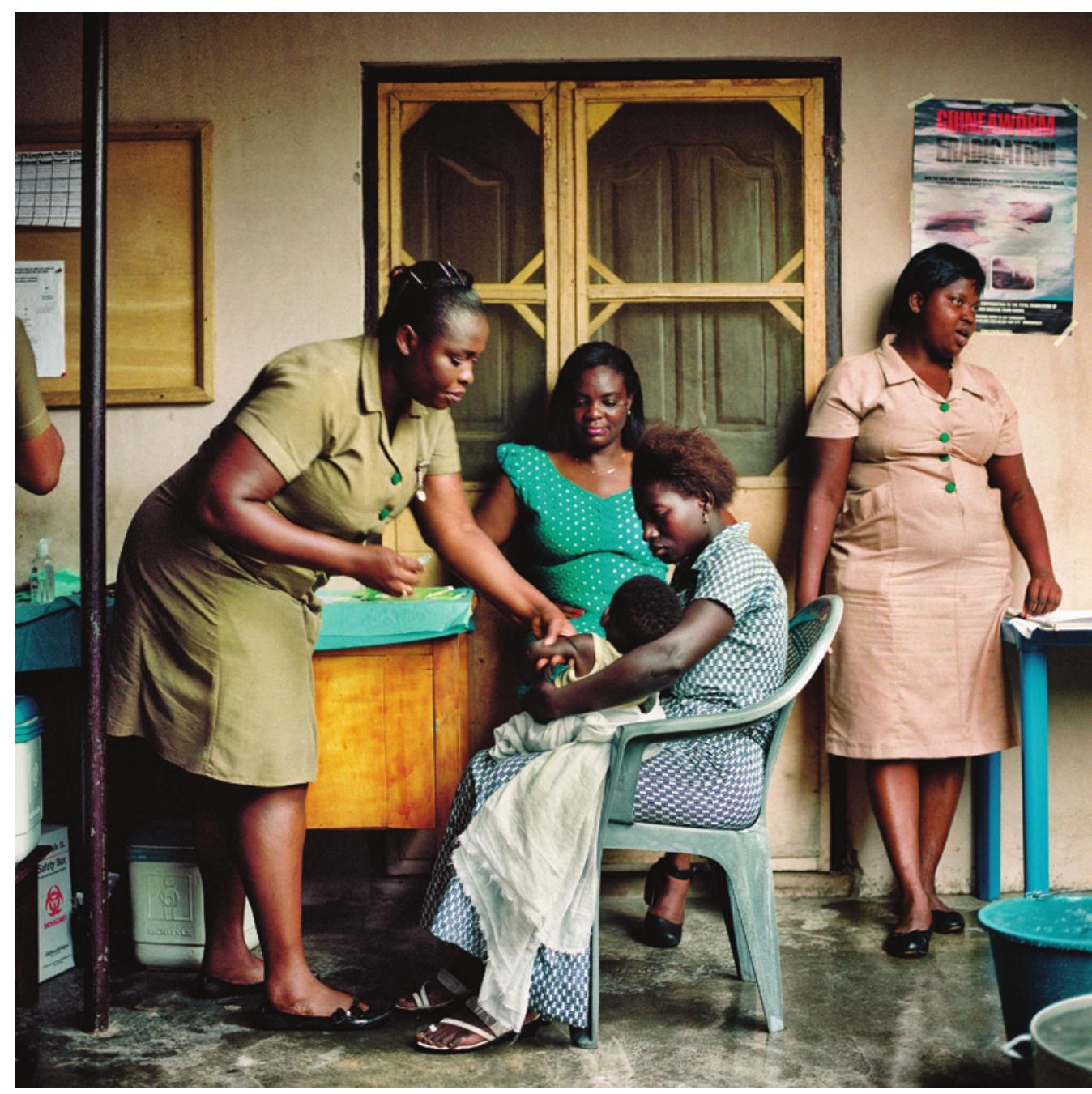

A nurse prepares to immunize a young child with pneumococcal and rotavirus vaccines in Ghana.

\section{VACCINES}

\section{Hidden bonus from vaccination}

\section{Immunization against pneumococcus in Africa also reduces levels of antibiotic resistance.}

\section{BY EWEN CALLAWAY}

$\mathrm{T}$

This summer, Eritrea, Côte d'Ivoire and Niger will join a growing list of coun-

tries where infants receive a vaccine to prevent pneumonia, meningitis and other deadly diseases caused by the pneumococcus bacterium (Streptococcus pneumoniae). Pneumonia is a leading killer of young children in low-income countries; vaccinations from 2010 to the end of this year are estimated to have averted 500,000 deaths, according to the GAVI Alliance in Geneva, an international organization that facilitates vaccination.

Data from South Africa also point to another benefit of vaccination: stemming a rising tide of antibiotic resistance in the developing world. The country's introduction of a pneumococcal conjugate vaccine (PCV) in 2009 has not only reduced the overall incidence of invasive pneumococcal disease by about two-thirds in infants (the age group vaccinated) and in adults, but has also reduced penicillin-resistant infections in both groups.

This is the first time such benefits have been observed outside the developed world. 